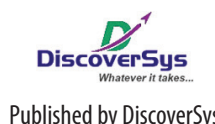

Published by DiscoverSys

\section{Nutritional status and associated factors in under-five children in Lembar Village West Lombok, Indonesia}

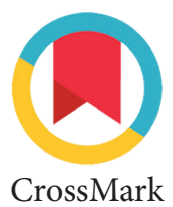

\author{
Widya Dwijayanti, ${ }^{1 *}$ Dewa Nyoman Wirawan, ${ }^{2}$ Luh Seri Ani, ${ }^{2}$ Ni Wayan Arya Utami ${ }^{2}$
}

\title{
ABSTRACT
}

Background and purpose: Studies on determinants of nutritional status in under-five children in Indonesia have been widely explored. However, most studies utilised only one out of three nutritional status indicators-weight/age, height/age, or weight/height. These studies also show inconsistent results. This present study aims to examine nutritional status in under-five children by using all three indicators and associated factors which include mother's characteristics, nutrition intake, and child's history of infectious diseases.

Methods: A cross-sectional study was conducted with a total sample of 100 under-five children who were randomly selected. Data on the mother's characteristics, nutrition intake, and child's history of infectious diseases were collected through structured interview with the mother, while data on nutritional status were obtained through measurements. Data were analysed using logistic regression to identify association between the nutritional status of under-five children with the mother's characteristics, nutrition intake, and child's history of infectious diseases.

Results: There was no association between the nutritional status of under-five children based on weight/age, height/age, and weight/ height with the mother's characteristics and nutrition intake. However, there was an association between the nutritional status of under-five children and child's history of infectious diseases with an adjusted odd ratio (AOR) $=41.0$ ( $95 \% \mathrm{Cl}: 12.10-139.14)$ for weight/age, AOR=3.52 (95\%Cl: $1.53-8.05)$ for height/age, and AOR=10.69 (95\%Cl: 1.31-87.11) for weight/height.

Conclusions: Child's history of infectious diseases is the only determinant associated with nutritional status in under-five children based on weight/ age, height/age, and weight/height. Prevention measures are required to prevent infection among children by improving environmental hygiene as well as providing prompt treatment for infectious diseases especially diarrhoea and upper-respiratory tract infection.
'STIKES Yarsi Mataram,

${ }^{2}$ Department of Public Health and Preventive Medicine Faculty of Medicine Udayana University
*Correspondence to:

Widya Dwijayanti, STIKES Yarsi Mataram

widya.dj91@gmail.com

Keywords: Nutritional status, infectious diseases, nutrition intake, mother's characteristics

Cite This Article: Dwijayanti, W., Wirawan, D.N., Ani, L.S., Utami, N.W.A. 2018. Nutritional status and associated factors in under-five children in Lembar Village West Lombok, Indonesia. Public Health and Preventive Medicine Archive 6(1): 17-25. D0I:10.15562/phpma.v6i1.4

\section{INTRODUCTION}

The Indonesia Basic Health Research (Riskesdas) 2013 showed that the prevalence of under-weight and stunting in under-five children were $19.6 \%$ and $37.2 \%$ respectively. ${ }^{1}$ The prevalence of under-weight in under-five children in West Nusa Tenggara Province was $25.7 \%$-ranked $9^{\text {th }}$ out of 18 provinces that have higher malnutrition prevalence than the national average. Furthermore, the prevalence of stunting in under-five children was $45.3 \%$-ranked 3 rd out of 20 provinces. ${ }^{1}$

The prevalence of under-weight in under-five children using weight/age indicator in West Lombok is $15.98 \%$. This figure is the second highest in West Nusa Tenggara Province. ${ }^{2}$ Jempatan Lembar Public Health Centre reported that the highest proportion of malnutrition was found in South Lembar Village with total cases of 48 out of 217 under-five children $(22.2 \%))^{3}$

Studies on nutritional status in under-five children and its determinants have been widely explored in Indonesia. However, these studies utilise only one out of three available indicators-weight/age, height/age, or weight/ height. These studies also show inconsistent results. A study in Tamamaung District, Makasar City, found a positive association between nutritional status in under-five children based on height/age and weight/ height with energy intake, but there was no association with protein intake. ${ }^{4}$ In contrast, a study in Semarang City using the same indicators found a positive correlation between nutritional status in under-five children and protein intake, but there was no association with energy intake. ${ }^{5}$ Another study in Kendari, South Sulawesi, found an association between nutritional status in under-five children based on weight/age indicator with a history of contracting infectious diseases. ${ }^{6}$ Other study exploring the Indonesia Basic Health Research (Riskesdas) 2007 data found an association between nutritional status in under-five children using weight/age, height/age, and weight/height indicators with diarrhoea. It also found an association between nutritional status in 
under-five children using weight/age and height/ age indicators with upper-respiratory infection. ${ }^{7}$

This present study aims to examine nutritional status in under-five children using weight/ age, height/age, and weight/height indicators and its association with the mother's characteristics, nutrition intake, and child's history of infectious diseases.

\section{METHODS}

A cross-sectional study was conducted in South Lembar Village, Lembar Sub-District, West Lombok District, West Nusa Tenggara Province. One hundred samples were recruited from all under-five children in South Lembar Village using systematic random sampling. Data on the mother's characteristics, nutrition intake, and child's history of infectious diseases were collected through a structured interview. Data on the nutritional status were obtained through direct measurements of weight and height. Interview and measurements were conducted in the respondent's house by the researcher and trained enumerators. Written informed consent was obtained before the interview and measurement. Anthropometry method was used to determine the nutritional status of under-five children based on indicators of weight/age, height/age, and weight/height. A digital weigher (Camry) was utilised to measure the weight of under-five children and a microtoise to measure the height. Age of the children was verified by comparing data obtained from interview and the growth chart card (KMS/Kartu Menuju Sehat). The under-five children were categorised as a stunted, under-weight, and thin child if indicators of height/age, weight/age, and weight/height with a Z-score $<-2 S D$. Data were analysed using STATA SE 12.1. Logistic regression was applied to examine the association between the nutritional status of under-five children with the mother's characteristics, nutrient intake, and child's history of infectious diseases. The study has obtained the ethical clearance from the Human Research Ethics Committee, Faculty of Medicine Mataram University.

\section{RESULTS}

Table 1 shows the distribution frequency of nutritional status, age, and gender of children, mother's characteristics (age, education, employment, income, parity, birth spacing, number of underfive children in the family, marital status, knowledge level, exclusive breastfeeding status), child's history of infectious diseases, access to healthcare facilities, environmental sanitation, and nutrient intake (energy, protein, fat, and carbohydrate).
The average age of the children is 32.42 months $(\mathrm{SD}=12.9)$ with more males than females. The proportion of stunting (48\%) is higher than underweight $(46 \%)$ and thin child (11\%). The average age of the mother is 28.85 years ( $\mathrm{SD}=6.1$ ). The majority of the mother are married, junior high school graduates, housewives, with income of IDR 600,000/ month and above, parity of less than 3 , a birth spacing period with the previous child of $\geq 24$ months, and the number of under-five children in the family $\leq 2$. The majority of mothers have a good knowledge regarding nutritional intake, utilise healthcare facilities and have adequate environmental sanitation. As many as $94 \%$ of respondents reported to exclusively breastfed their children. The proportion of children reported by their mother to have diarrhoea and upper respiratory tract infection within the last three months is $53 \%$. Using a 24 hours food recall, it was found that as many as $66 \%$ and $58 \%$ of our children consume less energy and carbohydrate than the recommended amount. The level of environmental sanitation was determined by measuring the distance between animal farm and the house and water source. The majority of respondents use a pipe water source and have an animal farm about 30 metres away from their house.

Multivariate analysis was employed using logistic regression to examine the association between the nutritional status in under-five children with several independent variables. All independent variables with $\mathrm{p}$ value $<0.25$ in the bivariate analysis were included in the multivariate analysis. Table 2 shows an association between under-weight children (weight/age) and a history of contracting diarrhoea or upper respiratory tract infections with $\mathrm{AOR}=41.0$ (95\%CI: 12.10-139.14). Similarly, Table 3 and 4 show an association between nutritional status based on height/age and weight/height with a history of contracting diarrhoea or upper respiratory tract infections with $\mathrm{AOR}=3.52$ (95\% CI: 1.53-8.05) and 10.69 (95\%CI: 1.31-87.11).

\section{DISCUSSION}

Our study shows as many as $46 \%$ and $48 \%$ of under-five children are underweight and stunted. Our study also shows as many as $11 \%$ of underfive children is thin (weight/height). This finding is higher than the provincial average of $25.7 \%$ for underweight children. ${ }^{1}$ Relatively the same prevalence for stunting was found when compared to the provincial average (45.3\%). ${ }^{1}$ Stunting was defined based on height/age without taking into account the weight variable. Our study found a higher proportion of thin children (11\%) when compared to the provincial average $(6.7 \%) .{ }^{1}$ It was suspected that the different proportions of underweight and 
Table 1 Characteristics of respondents and their children, nutrient intake, child's history of infectious diseases and nutritional status of under-five children

\begin{tabular}{|c|c|c|}
\hline Variables & $\mathbf{n}$ & $\%$ \\
\hline Age (month), mean \pm SD & $32.42 \pm 12.9$ & \\
\hline \multicolumn{3}{|l|}{ Gender } \\
\hline Male & 55 & 55.0 \\
\hline Female & 45 & 45.0 \\
\hline \multicolumn{3}{|l|}{ Weight/age } \\
\hline Normoweight & 54 & 54.0 \\
\hline Underweight & 46 & 46.0 \\
\hline \multicolumn{3}{|l|}{ Height/age } \\
\hline Normal & 52 & 52.0 \\
\hline Stunted & 48 & 48.0 \\
\hline \multicolumn{3}{|l|}{ Weight/height } \\
\hline Normal & 89 & 89.0 \\
\hline Thin child & 11 & 11.0 \\
\hline Age (mother, year), mean \pm SD & $28.85 \pm 6.1$ & \\
\hline \multicolumn{3}{|l|}{ Marital status } \\
\hline Married & 93 & 93.0 \\
\hline Divorced & 7 & 7.0 \\
\hline \multicolumn{3}{|l|}{ Education } \\
\hline Above junior high school & 26 & 26.0 \\
\hline Junior high school and below & 74 & 74.0 \\
\hline \multicolumn{3}{|l|}{ Employment } \\
\hline Employed & 14 & 14.0 \\
\hline Housewives & 86 & 86.0 \\
\hline \multicolumn{3}{|l|}{ Income } \\
\hline$\geq$ IDR. 600,000,- & 76 & 76.0 \\
\hline$<$ IDR 600,000,- & 24 & 24.0 \\
\hline \multicolumn{3}{|l|}{ Parity } \\
\hline$<3$ & 76 & 76.0 \\
\hline$\geq 3$ & 24 & 24.0 \\
\hline \multicolumn{3}{|l|}{ Birth spacing } \\
\hline$\geq 24$ months & 97 & 97.0 \\
\hline$<24$ months & 3 & 3.0 \\
\hline \multicolumn{3}{|c|}{ Number of under-five children in the family } \\
\hline$\leq 2$ & 98 & 98.0 \\
\hline$>2$ & 2 & 2.0 \\
\hline \multicolumn{3}{|l|}{ Knowledge } \\
\hline Good & 89 & 89.0 \\
\hline Poor & 11 & 11.0 \\
\hline
\end{tabular}

thin children between our study and the provincial average from The Indonesia Basic Health Research are due to the different tools used to measure body weight. Digital weight scale of Camry without calibration was used in this study while the Indonesia Basic Health Research used digital weight scale of Fesco with daily calibration.

An association was only found between nutritional status in under-five children using indicators of weight/age, height/age, and weight/height with a history of contracting diarrhoea and upper respiratory infections. Our finding is consistent with other study exploring the 2007 Indonesia Basic Health Research data which also found an association between nutritional status in under-five children (weight/age and height/age) with upper respiratory infections. ${ }^{7}$ A study in Padang found a significant correlation $(\mathrm{p}=0.001)$ between nutritional status in under-five children and pneumonia. ${ }^{8}$ However, a study in Lolayan Subdistrict, Bolaang Mongondow District, Manado found no significant association between nutritional status in under-five children based on indicators of weight/age, height/age, and weight/height with infectious diseases (upper respiratory infection, diarrhoea, or pneumonia). ${ }^{9}$

Our study shows no significant association between nutritional status in under-five children with nutrient intake. This finding is consistent with another study in Tamamaung Village, Makasar City which found no correlation between nutritional status in under-five children (height/age) with protein intake. It also found no association between nutritional status (weight/height) among under-five children with energy and protein intakes. ${ }^{4} \mathrm{~A}$ study in Menduran Grobogan Village, Semarang City also found no association between nutritional status in under-five children (height/age and weight/height) with energy intake. However, they found an association between nutritional status among under-five children (weight/height) with protein intake. ${ }^{5}$

A study in Nyogan, Muaro District, Jambi Province found an association between nutritional status in under-five children (weight/age, height/ age, and weight/height) with energy and protein intakes $(\mathrm{p}<0.05) .{ }^{10}$ This study used a food recall 24 hours for consecuted three days to measure nutrient intake. ${ }^{10}$ In our study, though we also used a food recall 24 hours to measure nutrient intake, we only recorded it for one day.

Our study found no association between nutritional status among under-five children with the mother's characteristics (age, education 
Table 1 Characteristics of respondents and their children, nutrient intake, child's history of infectious diseases and nutritional status of under-five children

\begin{tabular}{|c|c|c|}
\hline Variables & $\mathbf{n}$ & $\%$ \\
\hline \multicolumn{3}{|c|}{ Access to healthcare } \\
\hline Yes & 86 & 86.0 \\
\hline No & 14 & 14.0 \\
\hline \multicolumn{3}{|c|}{ Environmental sanitation } \\
\hline Good & 65 & 65.0 \\
\hline Poor & 35 & 35.0 \\
\hline \multicolumn{3}{|c|}{ Exclusive breastfeeding } \\
\hline Yes & 94 & 94.0 \\
\hline No & 6 & 6.0 \\
\hline \multicolumn{3}{|c|}{ History of infectious diseases } \\
\hline No & 47 & 47.0 \\
\hline Yes & 53 & 53.0 \\
\hline \multicolumn{3}{|c|}{ Nutrient intake } \\
\hline Good & 34 & 34.0 \\
\hline Poor & 66 & 66.0 \\
\hline \multicolumn{3}{|c|}{ Protein intake } \\
\hline Good & 76 & 76.0 \\
\hline Poor & 24 & 24.0 \\
\hline \multicolumn{3}{|l|}{ Fat intake } \\
\hline Good & 94 & 94.0 \\
\hline Poor & 6 & 6.0 \\
\hline \multicolumn{3}{|c|}{ Carbohydrate intake } \\
\hline Good & 42 & 42.0 \\
\hline Poor & 58 & 58.0 \\
\hline Total & 100 & 100.00 \\
\hline
\end{tabular}

Table 2 Association between the nutritional status of under-five children (weight/age) with mother's characteristics, nutrient intake, and child's history of infectious diseases

\begin{tabular}{|c|c|c|c|c|c|}
\hline \multirow[b]{2}{*}{ Variables } & \multicolumn{2}{|c|}{ Bivariate } & \multicolumn{3}{|c|}{ Multivariate } \\
\hline & $\begin{array}{c}\text { Normoweight } \\
\text { n (\%) }\end{array}$ & $\begin{array}{c}\text { Underweight } \\
\text { n (\%) }\end{array}$ & p value & AOR & $95 \% \mathrm{Cl}$ \\
\hline \multicolumn{6}{|l|}{ Age (mother) } \\
\hline$>35$ years & $5(38.5)$ & $8(61.5)$ & 0.228 & 0.71 & $0.13-3.90$ \\
\hline$\leq 35$ years & $49(56.3)$ & $38(43.7)$ & & & \\
\hline \multicolumn{6}{|l|}{ Education } \\
\hline Above junior high school & $16(61.5)$ & $10(38.5)$ & 0.370 & & \\
\hline Junior high school and below & $38(51.3)$ & $36(48.7)$ & & & \\
\hline \multicolumn{6}{|l|}{ Employment } \\
\hline Employed & $6(42.9)$ & $8(57.1)$ & 0.367 & & \\
\hline Housewives & $48(55.8)$ & $38(44.2)$ & & & \\
\hline \multicolumn{6}{|l|}{ Income } \\
\hline$\geq$ IDR 600,000,- & $42(55.3)$ & $34(44.7)$ & 0.652 & & \\
\hline$<$ IDR 600,000,- & $12(50.0)$ & $12(50.0)$ & & & \\
\hline
\end{tabular}


Table 2 Association between the nutritional status of under-five children (weight/age) with mother's characteristics, nutrient intake, and child's history of infectious diseases

\begin{tabular}{|c|c|c|c|c|c|}
\hline \multirow[b]{2}{*}{ Variables } & \multicolumn{2}{|c|}{ Bivariate } & \multicolumn{3}{|c|}{ Multivariate } \\
\hline & $\begin{array}{c}\text { Normoweight } \\
\text { n (\%) }\end{array}$ & $\begin{array}{c}\text { Underweight } \\
\text { n (\%) }\end{array}$ & p value & AOR & $95 \% \mathrm{Cl}$ \\
\hline \multicolumn{6}{|l|}{ Parity } \\
\hline$<3$ & $39(51.3)$ & $37(48.7)$ & 0.338 & & \\
\hline$\geq 3$ & $15(62.5)$ & $9(37.5)$ & & & \\
\hline \multicolumn{6}{|l|}{ Birth spacing } \\
\hline$\geq 24$ months & $52(53.6)$ & $45(46.4)$ & 0.655 & & \\
\hline$<24$ months & $2(66.7)$ & $1(33.3)$ & & & \\
\hline \multicolumn{6}{|c|}{ Number of under-five children } \\
\hline$\leq 2$ & $53(54.1)$ & $45(45.9)$ & 0.909 & & \\
\hline$>2$ & $1(50.0)$ & $1(50.0)$ & & & \\
\hline \multicolumn{6}{|l|}{ Marital status } \\
\hline Married & $49(52.7)$ & $44(47.3)$ & 0.337 & & \\
\hline Divorced & $5(71.4)$ & $2(28.6)$ & & & \\
\hline \multicolumn{6}{|l|}{ Knowledge } \\
\hline Good & $50(56.2)$ & $39(43.8)$ & 0.213 & 2.57 & $0.38-17.06$ \\
\hline Poor & $4(36.4)$ & $7(63.6)$ & & & \\
\hline \multicolumn{6}{|c|}{ Exclusive breastfeeding } \\
\hline Yes & $52(55.3)$ & $42(44.7)$ & 0.295 & 41.0 & $12.10-139.14$ \\
\hline No & $2(33.3)$ & $4(66.7)$ & & & \\
\hline \multicolumn{6}{|c|}{ History of infectious diseases } \\
\hline No & $43(91.5)$ & $4(8.5)$ & 0.000 & & \\
\hline Yes & $11(20.8)$ & $42(79.2)$ & & & \\
\hline \multicolumn{6}{|c|}{ Access to healthcare } \\
\hline Yes & $46(53.5)$ & $40(46.5)$ & 0.799 & & \\
\hline No & $8(57.1)$ & $6(42.9)$ & & & \\
\hline \multicolumn{6}{|c|}{ Environmental sanitation } \\
\hline Good & $35(53.9)$ & $30(46.1)$ & 0.966 & & \\
\hline Poor & $19(54.3)$ & $16(45.7)$ & & & \\
\hline \multicolumn{6}{|l|}{ Energy intake } \\
\hline Good & $20(58.8)$ & $14(41.2)$ & 0.487 & & \\
\hline Poor & $34(51.5)$ & $32(48.5)$ & & & \\
\hline \multicolumn{6}{|c|}{ Protein intake } \\
\hline Good & $42(55.3)$ & $34(44.7)$ & 0.652 & & \\
\hline Poor & $12(50.0)$ & $12(50.0)$ & & & \\
\hline \multicolumn{6}{|l|}{ Fat intake } \\
\hline Good & $51(54.3)$ & $43(42.7)$ & 0.839 & & \\
\hline Poor & $3(50.0)$ & $3(50.0)$ & & & \\
\hline \multicolumn{6}{|c|}{ Carbohydrate intake } \\
\hline Good & $20(47.6)$ & $22(53.4)$ & 0.276 & & \\
\hline Poor & $34(58.6)$ & $24(41.4)$ & & & \\
\hline
\end{tabular}


Table 3 Association between the nutritional status of under-five children (height/age) with mother's characteristics, nutrient intake, and child's history of infectious diseases

\begin{tabular}{|c|c|c|c|c|c|}
\hline \multirow[b]{2}{*}{ Variables } & \multicolumn{2}{|c|}{ Bivariate } & & \multicolumn{2}{|c|}{ Multivariate } \\
\hline & $\begin{array}{c}\text { Normal } \\
\text { n (\%) }\end{array}$ & $\begin{array}{c}\text { Stunted } \\
\mathbf{n}(\%)\end{array}$ & p value & AOR & $95 \% \mathrm{Cl}$ \\
\hline \multicolumn{6}{|l|}{ Age (mother) } \\
\hline$>35$ years & $5(38.5)$ & $8(61.5)$ & 0.295 & & \\
\hline$\leq 35$ years & $47(54.0)$ & $40(46.0)$ & & & \\
\hline \multicolumn{6}{|l|}{ Education } \\
\hline Above junior high school & $15(57.7)$ & $11(42.3)$ & 0.499 & 0.48 & $0.13-1.67$ \\
\hline Junior high school and below & $37(50.0)$ & $37(50.0)$ & & & \\
\hline \multicolumn{6}{|l|}{ Employment } \\
\hline Employed & $5(35.7)$ & $9(64.3)$ & 0.188 & & \\
\hline Housewives & $47(54.6)$ & $39(45.4)$ & & & \\
\hline \multicolumn{6}{|l|}{ Income } \\
\hline$\geq$ IDR $600,000,-$ & $40(52.6)$ & $36(47.4)$ & 0.822 & & \\
\hline$<$ IDR 600,000,- & $12(50.0)$ & $12(50.0)$ & & & \\
\hline \multicolumn{6}{|l|}{ Parity } \\
\hline$<3$ & $41(54.0)$ & $35(46.0)$ & 0.488 & & \\
\hline$\geq 3$ & $11(45.9)$ & 13(54.1) & & & \\
\hline \multicolumn{6}{|l|}{ Birth spacing } \\
\hline$\geq 24$ months & $51(52.6)$ & $46(47.4)$ & 0.511 & & \\
\hline$<24$ months & $1(33.3)$ & $2(66.7)$ & & & \\
\hline \multicolumn{6}{|l|}{ Number of under-five children } \\
\hline$\leq 2$ & $51(52.0)$ & $47(48.0)$ & 0.954 & & \\
\hline$>2$ & $1(50.0)$ & $1(50.0)$ & & & \\
\hline \multicolumn{6}{|l|}{ Marital status } \\
\hline Married & $46(49.5)$ & $47(50.5)$ & 0.064 & 0.21 & $0.02-1.99$ \\
\hline Divorced & $6(85.7)$ & $1(14.3)$ & & & \\
\hline \multicolumn{6}{|l|}{ Knowledge } \\
\hline Good & $45(50.6)$ & $44(49.4)$ & 0.413 & & \\
\hline Poor & $7(63.6)$ & $4(36.4)$ & & & \\
\hline \multicolumn{6}{|l|}{ Exclusive breastfeeding } \\
\hline Yes & $51(54.3)$ & $43(45.7)$ & 0.074 & 5.66 & $0.59-53.76$ \\
\hline No & $1(16.7)$ & $5(83.3)$ & & & \\
\hline \multicolumn{6}{|l|}{ History of infectious diseases } \\
\hline No & $32(68.1)$ & $15(31.9)$ & 0.002 & 3.52 & $1.53-8.05$ \\
\hline Yes & $20(37.7)$ & $33(62.3)$ & & & \\
\hline \multicolumn{6}{|l|}{ Access to healthcare } \\
\hline Yes & $43(50.0)$ & $43(50.0)$ & 0.321 & & \\
\hline No & $9(64.3)$ & $5(35.7)$ & & & \\
\hline \multicolumn{6}{|l|}{ Environmental sanitation } \\
\hline Good & $34(52.3)$ & $31(47.7)$ & 0.933 & & \\
\hline Poor & $18(51.4)$ & $17(48.6)$ & & & \\
\hline
\end{tabular}


Table 3 Association between the nutritional status of under-five children (height/age) with mother's characteristics, nutrient intake, and child's history of infectious diseases

\begin{tabular}{lccccc}
\hline & \multicolumn{2}{c}{ Bivariate } & & Multivariate \\
\cline { 2 - 6 } Variables & $\begin{array}{c}\text { Normal } \\
\mathbf{n}(\%)\end{array}$ & $\begin{array}{c}\text { Stunted } \\
\mathbf{n}(\%)\end{array}$ & p value & AOR & $\mathbf{9 5 \% C l}$ \\
\hline Energy intake & & & & & \\
$\quad$ Good & $15(44.1)$ & $19(55.9)$ & 0.257 & & \\
$\quad$ Poor & $37(56.1)$ & $29(43.9)$ & & & \\
Protein intake & & & & & \\
$\quad$ Good & $39(51.3)$ & $37(48.7)$ & 0.807 & \\
$\quad$ Poor & $13(54.2)$ & $11(45.8)$ & & \\
Fat intake & & & & & \\
$\quad$ Good & $48(51.1)$ & $46(48.9)$ & 0.458 & & \\
$\quad$ Poor & $4(66.7)$ & $2(33.3)$ & & & \\
Carbohydrate intake & & & & & \\
$\quad$ Good & $19(45.2)$ & $23(54.8)$ & $0.28-1.70$ \\
$\quad$ Poor & $33(56.9)$ & $25(43.1)$ & & & \\
\hline
\end{tabular}

Table 4 Association between the nutritional status of under-five children (weight/height) with mother's characteristics, nutrient intake, and child's history of infectious diseases

\begin{tabular}{|c|c|c|c|c|c|}
\hline \multirow[b]{2}{*}{ Variables } & \multicolumn{2}{|c|}{ Bivariate } & \multirow[b]{2}{*}{$p$ value } & \multicolumn{2}{|c|}{ Multivariate } \\
\hline & $\begin{array}{c}\text { Normal } \\
\text { n (\%) }\end{array}$ & $\begin{array}{l}\text { Thin } \\
\mathrm{n}(\%)\end{array}$ & & AOR & $95 \% \mathrm{Cl}$ \\
\hline \multicolumn{6}{|l|}{ Age (mother) } \\
\hline$>35$ years & $10(76.9)$ & $3(23.1)$ & 0.136 & 0.82 & $0.13-5.17$ \\
\hline$\leq 35$ years & $79(90.8)$ & $8(9.2)$ & & & \\
\hline \multicolumn{6}{|l|}{ Education } \\
\hline Above junior high school & $26(100.0)$ & $0(0.0)$ & 0.037 & & \\
\hline Junior high school and below & $63(85.1)$ & $11(14.9)$ & & & \\
\hline \multicolumn{6}{|l|}{ Employment } \\
\hline Employed & $13(92.9)$ & $1(7.1)$ & 0.619 & & \\
\hline Housewives & $76(88.4)$ & $10(11.6)$ & & & \\
\hline \multicolumn{6}{|l|}{ Income } \\
\hline$\geq$ IDR 600,000,- & $67(88.2)$ & $9(11.8)$ & 0.632 & & \\
\hline$<$ IDR 600,000,- & $22(91.7)$ & $2(8.3)$ & & & \\
\hline \multicolumn{6}{|l|}{ Parity } \\
\hline$<3$ & $69(90.8)$ & $7(9.2)$ & 0.309 & & \\
\hline$\geq 3$ & $20(83.3)$ & $4(16.7)$ & & & \\
\hline \multicolumn{6}{|l|}{ Birth spacing } \\
\hline$\geq 24$ months & $87(89.7)$ & $10(10.3)$ & 0.209 & 0.23 & $0.04-1.17$ \\
\hline$<24$ months & $2(66.7)$ & $1(33.3)$ & & & \\
\hline \multicolumn{6}{|l|}{ Number of under-five children } \\
\hline$\leq 2$ & $87(88.8)$ & $11(11.2)$ & 0.616 & & \\
\hline$>2$ & $2(100.0)$ & $0(00.0)$ & & & \\
\hline
\end{tabular}


Table 4 Association between the nutritional status of under-five children (weight/height) with mother's characteristics, nutrient intake, and child's history of infectious diseases

\begin{tabular}{|c|c|c|c|c|c|}
\hline \multirow[b]{2}{*}{ Variables } & \multicolumn{2}{|c|}{ Bivariate } & & \multicolumn{2}{|c|}{ Multivariate } \\
\hline & $\begin{array}{c}\text { Normal } \\
\text { n (\%) }\end{array}$ & $\begin{array}{l}\text { Thin } \\
\text { n (\%) }\end{array}$ & p value & AOR & $95 \% \mathrm{Cl}$ \\
\hline \multicolumn{6}{|c|}{ Marital status } \\
\hline Married & $83(89.2)$ & $10(10.8)$ & 0.773 & & \\
\hline Divorced & $6(85.7)$ & $1(14.3)$ & & & \\
\hline \multicolumn{6}{|l|}{ Knowledge } \\
\hline Good & $81(91.0)$ & $8(9.0)$ & 0.067 & 5.13 & $0.86-30.49$ \\
\hline Poor & $8(72.7)$ & $3(27.3)$ & & & \\
\hline \multicolumn{6}{|c|}{ Exclusive breastfeeding } \\
\hline Yes & $83(88.3)$ & $11(11.7)$ & 0.374 & & \\
\hline No & $6(100.0)$ & $0(00.0)$ & & & \\
\hline \multicolumn{6}{|c|}{ History of infectious diseases } \\
\hline No & $46(97.9)$ & $1(2.1)$ & 0.008 & 10.69 & $1.31-87.11$ \\
\hline Yes & $43(81.1)$ & $10(18.9)$ & & & \\
\hline \multicolumn{6}{|c|}{ Access to healthcare } \\
\hline Yes & $76(88.4)$ & $10(11.6)$ & 0.619 & & \\
\hline No & $13(92.9)$ & $1(7.1)$ & & & \\
\hline \multicolumn{6}{|c|}{ Environmental sanitation } \\
\hline Good & $56(86.1)$ & $9(13.9)$ & 0.215 & 0.35 & $0.06-1.93$ \\
\hline poor & $33(94.3)$ & $2(5.7)$ & & & \\
\hline \multicolumn{6}{|c|}{ Energy intake } \\
\hline Good & $32(94.1)$ & $2(5.9)$ & 0.240 & 1.40 & $0.24-8.02$ \\
\hline Poor & $57(86.4)$ & $9(13.6)$ & & & \\
\hline \multicolumn{6}{|c|}{ Protein intake } \\
\hline Good & $69(92.0)$ & $6(8.0)$ & 0.097 & & \\
\hline Poor & $20(80.0)$ & $5(20.0)$ & & & \\
\hline \multicolumn{6}{|l|}{ Fat intake } \\
\hline Good & $84(89.4)$ & $10(10.6)$ & 0.674 & & \\
\hline Poor & $5(83.3)$ & $1(16.7)$ & & & \\
\hline \multicolumn{6}{|c|}{ Carbohydrate intake } \\
\hline Good & $38(90.5)$ & $4(9.5)$ & 0.688 & & \\
\hline Poor & $51(87.9)$ & $7(12.1)$ & & & \\
\hline
\end{tabular}

level, employment, income, parity, birth spacing, and number of under-five children in the family). Our finding is consistent with other studies in Ajung Sub-district, Jember District ${ }^{11}$ and Kao Sub-district North Halmahera District. ${ }^{12}$ These studies found no significant correlation between nutritional status in under-five children and the number of under-five children in the family. Furthermore, secondary analysis of the 2007 and 2013 Indonesia Basic Health Research for Lebak
District and Tangerang City revealed that there is no significant association between nutritional status in under-five children (weight/age, height/ age, and weight/height) with the employment of their parent. ${ }^{13}$ Another study in Manado also found no correlation between nutritional status among under-five children with the mother's characteristics (age and employment). However it showed an association between education level and parity. ${ }^{14}$ In North Halmahera, the nutritional 
status of under-five children is associated with birth spacing. ${ }^{12}$

To reduce the prevalence of underweight, stunting, and thin child among under-five children, prevention measures especially improving environment sanitation and prompt treatment for any infectious diseases should be enhanced. The main limitations of our study include the uncalibrated weight scale and using the food recall 24 hours only for one day. In addition, we only cover the limited area in West Nusa Tenggara Province leading to the limited generalizability of our findings to the wider population.

\section{CONCLUSION}

The nutritional status of under-five children based on weight/age, height/age, and weight/height indicators is associated with diarrhoea and upper respiratory infections, but there is no significant association with nutrient intake and the mother's characteristics.

\section{ACKNOWLEDGEMENT}

We would like to extend our gratitude to all respondents, the Head of Department of Health of West Lombok District, the Head of Jembatan Kembar Public Health Centre and midwives in South Lembar Village for supporting this study.

\section{REFERENCES}

1. Health Research and Development Agency, Ministry of Health of Indonesia. Riset Kesehatan Dasar (Riskesdas) 2013 [The Indonesia Basic Health Research 2013]. 2013.

2. Department of Health of West Nusa Tenggara Province. Profil Kesehatan Provinsi Nusa Tenggara Barat [Health Profile of West Nusa Tenggara Province]. 2015.

3. Jembatan Kembar Public Health Centre. Laporan hasil rekapan status gizi balita Pekan Imunisasi Nasional tahun 2016 [Report on nutritional status of under-five children in National Immunisation Week Program 2016]. 2016

4. Muchlis N, Hadju V, Jafar N. Hubungan asupan energi dan protein dengan status gizi balita di Kelurahan Tamamaung [Association between energy and protein intakes and nutritional status of under-five children in Tamamaung Village]. Hasanuddin Student Journal 2007; 1: 1-8.
5. Febrindari AP, Nuryanto. Hubungan asupan energi, protein, seng dan kejadian infeksi kecacingan status gizi anak umur 12-36 bulan [Correlation between energy, protein, and zinc intakes with worm infestation and nutritional status among children aged 12-36 months]. Journal Nutritional College 2016; 5: 353-359.

6. Lestari J, Majid R, Sakka A, et al. Eating parenting relations, history of infectious diseases and access of health care with nutritional status in children under five in coastal areas. International Journal Science 2015; 1: 2395-3470.

7. Hidayat TS, Fuada N. Hubungan sanitasi lingkungan, morbiditas dan status gizi balita di Indonesia [Association between environment sanitation, morbidity, and nutritional status among under-five children in Indonesia]. Penelitian Gizi Masyarahat 2011; 34: 104-113.

8. Nurnajiah M, Rusdi, Desmawati. Hubungan status gizi dengan derajat pneumonia pada balita di RS. Dr. M. Djamil Padang [Association between nutritional status and pneumonia among under-five children in Dr. M. Djamil Padang Hospital]. Jurnal Kesehatan Andalas 2016; 5: 250-255.

9. Putri MS, Kapantow N, Kawengian S. Hubungan antara riwayat penyakit infeksi dengan status gizi pada anak batita di Desa Mopusi Kecamatan Lolayan Kabupaten Bolaang Mongondow [Association between history of contracting infectious diseases and nutritional status among under-five children in Mopusi Village Lolayan Sub-district, Bolaang Mongondow District]. Jurnal E-Biomedik 2015; 3: 576-580

10. Erni, Juffrie M, Rialihanto M. Pola makan, asupan zat gizi, dan status gizi anak balita Suku Anak Dalam di Nyogan Kabupaten Muaro Jambi Provinsi Jambi [Diet pattern, nutrient intake, and nutritional status among under-five children in Anak Dalam Tribe, Nyogan, Muaro District - Jambi Province]. Jurnal Gizi Klinis Indonesia 2008; 5: 84-90.

11. Indarti Y. Hubungan status ekonomi keluarga dengan status gizi balita di Kecamatan Ajung Kabupaten Jember Tahun 2016 [Association between socio-economic status and nutritional status of under-five children in Ajung Subdistrict, Jember District 2016]. Fenomena 2016; 15: 149-162.

12. Karundeng LR, Kundre R. Status gizi balita di Puskesmas Kao Halmahera Utara [Nutritional status in under-five children in Kao Public Health Centre, North Halmahera]. Jurnal Keperawatan 2015; 3:1-9.

13. Sukoco NEW, Pambudi J, Herawati MH. Hubungan status gizi anak balita dengan orang tua bekerja [Association between nutritional status of under-five children and employment status of their parent]. Buletin Penelitian Sistem Kesehatan 2015; 18: 387-397.

14. Labada A, Ismanto AY, Kundre R. Hubungan karakteristik ibu dengan status gizi balita yang berkunjung di Puskesmas Bahu Manado [Association between the mother's characteristics and nutritional status of underfive children in Bahu Public Health Centre, Manado]. eJurnal Keperawatan 2016; 4: 1-8.

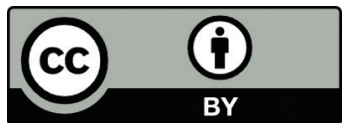

This work is licensed under a Creative Commons Attribution 